\title{
A Symmetry-Based Length Model For Describing The Hypersonic Boundary Layer Transition On A Slender Cone At Moderate Incidence
}

\section{Weitao Bi}

Peking University

\section{Zhou Wei}

Peking University

Kexin Zheng

Peking University

Zhensu She ( $\nabla$ she@pku.edu.cn )

Peking University https://orcid.org/0000-0001-7001-9995

\section{Research Article}

Keywords: Hypersonic boundary layer transition, Transition model, Cone, Symmetry-based length model

Posted Date: February 9th, 2022

DOI: https://doi.org/10.21203/rs.3.rs-1286457/v1

License: (c) This work is licensed under a Creative Commons Attribution 4.0 International License. Read Full License 


\title{
A symmetry-based length model for describing the hypersonic boundary layer transition on a slender cone at moderate incidence
}

\author{
Wei-Tao Bi, Zhou Wei, Ke-Xin Zheng and Zhen-Su She*
}

\footnotetext{
${ }^{*}$ Correspondence: she@pku.edu.cn State Key Laboratory for Turbulence and Complex Systems and Department of mechanics and Engineering Science, College of Engineering, Peking University, Beijing 100871, P. R. China Full list of author information is available at the end of the article
}

\begin{abstract}
The hypersonic boundary layer $(\mathrm{HBL})$ transition on a slender cone at moderate incidence has been studied via a symmetry-based length model: the SED-SL model. The SED-SL specifies an analytic stress length function (which defines the eddy viscosity) parameterizing a two-dimensional multi-regime structure characterizing the laminar-turbulence transition. Previous studies show accurate predictions by the SED-SL for airfoil flows at different subsonic Mach numbers, Reynolds numbers and angles of attack, especially for the drag coefficient achieving a small error of a few counts. Here, we report a successful extension for computing transitional flows on a $7^{\circ}$ half-angle straight cone at Mach numbers 6 and 7 and angles of attack from $0^{\circ}$ to $6^{\circ}$. It is shown that, by properly setting the multi-regime HBL structure with only three physical parameters (i.e. a transition center location, a turbulent HBL near-wall eddy length, and a transition width coefficient quantifying overshoot strength), the SED-SL is able to predict correctly the heat flux distribution on the whole cone surface, with great accuracy compared with wind tunnel data. The good predictions cover a range of flow parameters and persist in different instability mechanisms (which vary from the windward to the leeward sides of the cone). We conclude that a unified transition model is found for describing the transitional HBLs, with the potential to lead a new generation of turbulence model that is simultaneously of computation simplicity, sound physics and greater accuracy.
\end{abstract}

Keywords: Hypersonic boundary layer transition; Transition model; Cone; Symmetry-based length model

\section{Introduction}

Hypersonic boundary layer (HBL) transition has a crucial impact on the performance of high-speed flying vehicles and has been the focus of many theoretical, experimental and numerical studies during the past several decades. Compared to low-speed flows, HBL transition is subjected to more instability mechanisms with more influential factors, making it a tremendous challenge to both scientists and engineers. Anderson [1] has concluded that the transition onset Reynolds number (Re) of HBL depends on nearly twenty factors in general. For flow over a straight cone [2], specifically, the most relevant factors include the free-stream unit Re, Mach number (Ma), total and wall temperatures, free-stream noise level, angle of attack (AoA), nose-tip bluntness, surface roughness, and half angle of the cone, etc. Formulating a model describing all these effects on the HBL transition has thus been a long-lasting research subject for many experimentalists and engineers. Unfortu- 
nately, very few results are satisfactory, which cover limited parameter ranges for a small number of factors, with limited accuracy, owing to the complexity from the interplay of multiple factors [2] and from the variation of influential patterns in wide factor ranges (e.g. for nose-tip bluntness [3]). Since nearly all transition models include a correlation equation to define the transition onset location $[4,5]$, which is poorly predicted in general, an obvious limitation of the existed hypersonic transition models is that it usually gives insufficient and even undetermined prediction accuracies $[6,7]$. Furthermore, HBLs are generally governed by varying transition mechanisms $[2,8]$, depending on the location of surface areas. Taking the HBL on an inclined straight cone as an example, the Mack second mode is widely considered to be the dominant instability mode on the windward surface, the cross-flow mode on the lateral side, and the streamwise vortex mode on the leeward center of the cone $[9,10,11]$. The varying transition mechanisms result in enormous and ever increasing complexities in modeling and simulating HBL transitions [12, 13], which calls for a reflection: whether one should always rely on a correct description of the transition mechanism for the foundation of a transition model. One perhaps needs to discover a universal principle governing transitional boundary layers, and to work out a more efficient transitional model, rather than keeping proposing more and more complex version of the popular intermittency-factor-based transition models. We attempt to develop a new approach.

To begin, we propose a notion of "similarity structure" of boundary layers, which ensures a similarity of the flow for varying Re and Ma. We further introduce a notion of "order function" as the similarity variable to display the right symmetry of the "similarity structure". Specifically, for boundary layers, the most important symmetry is the symmetry under a dilation transformation from the wall in the wall-normal direction and that from the leading edge in the streamwise direction. And the similarity variable is a stress length (SL) that characterizes the size of eddies most relevant to the momentum transport. In the so-called structural ensemble dynamics (SED) theory $[14,15]$, it is postulated that a wall-normally fourlayer structure corresponding to the viscous sublayer, buffer layer, log-layer and bulk flow, is the similarity structure of turbulent boundary layer (TBL). The stress length function displays a unique power law with wall distance within each layer, as well as a universal transition between adjacent layers, which are derived from a novel Lie-group analysis of the momentum and energy balance equations, and validated in detail for the canonical wall-bounded turbulence (i.e. channel, circular pipe, and zero-pressure-gradient flat-plate TBL) [14,15]. It has been shown that the mean-flow profiles of the canonical TBL are thus predicted, for the first time, over the whole boundary layer thickness and for all Re, at an unprecedented accuracy compared to direct numerical simulation and experimental data $[14,16,17]$.

More recently, a streamwise three-layer structure (corresponding to the laminar, transitional and fully-developed TBL states, respectively) is suggested for the stress length function to describe transitional boundary layers, which yields a new algebraic transition model called SED-SL [18], since the SL fully specifies the twodimensional eddy viscosity distribution throughout the transition process. Application to several airfoil flows (e.g. NACA0012 and RAE2822) at different subsonic Ma, Re and AoA, yields an unprecedented accuracy (up to a few counts) in the 
drag prediction [19], indicating the validity of the concept of the "similarity structure", and pointing out a valuable new direction for turbulence model construction. It is thus anticipated that this similarity-structure-based transition model captures universal features of the transition processes without showing intricate dependency on the influential factor variation and involving specific instability mechanism that causes the transition. We present here a preliminary study to extend the SED-SL to the HBL transition of straight cone, to further validate the merit of the model.

Here, we apply the SED-SL to compute the transitional flows on a $7^{\circ}$ half-angle straight cone at Ma 6 and 7 and AoA from $0^{\circ}$ to $6^{\circ}$. It is shown that, by properly setting the two-dimensional multi-regime structure of the HBL over the cone through only three parameters of clear physical meaning (i.e. a transition center, an after-transition near-wall eddy length, and a transition width coefficient related to overshoot strength), the SED-SL correctly describes the heat flux distribution on the whole cone surface, in excellent agreement with the wind tunnel data. We demonstrate a simple adaptation of the SED-SL to different flow parameters (e.g. Ma, AoA, free-stream disturbance), and to different instability mechanisms (which vary with AoA and the circumferential angle of the cone). Thus, we have proposed a unified description for the HBL transition under the change of several influential factors and transition mechanisms, which paves a way to a new algebraic transition model for engineering applications that is simultaneously of computation simplicity, sound physics and promising accuracy.

The paper is organized as follows. Section 2 describes the SED-SL model and the computational setup. Section 3 presents the results of computation, which validate our predictions for different flow cases. Section 4 is devoted to discussion and conclusion.

\section{Theory}

The compressible Reynolds-averaged Navier-Stokes (RANS) equations, which express the mean conservation of mass, momentum and energy, under the thin-layer and Boussinesq approximations and assuming constant turbulent Prandtl number, are written as follows:

$$
\begin{aligned}
& \frac{\partial \rho}{\partial t}+\frac{\partial}{\partial x_{j}}\left(\rho U_{j}\right)=0, \\
& \frac{\partial \rho U_{i}}{\partial t}+\frac{\partial}{\partial x_{j}}\left(\rho U_{i} U_{j}\right)=-\frac{\partial p}{\partial x_{i}}+\frac{\partial \sigma_{i j}}{\partial x_{j}}, \\
& \frac{\partial \rho E}{\partial t}+\frac{\partial}{\partial x_{j}}\left(\rho U_{j} H\right)=\frac{\partial}{\partial x_{j}}\left(U_{i} \sigma_{i j}\right)-\frac{\partial}{\partial x_{j}}\left[C_{p}\left(\frac{\mu}{\operatorname{Pr}}+\frac{\mu_{t}}{\operatorname{Pr}_{t}}\right) \frac{\partial T}{\partial x_{j}}\right],
\end{aligned}
$$

where $\rho$ is fluid density, $U_{j}$ is the $j$-th component of mean velocity and $x_{j}$ represents the Cartesian coordinate. $p=\rho R T$ is the static pressure ( $R$ is the gas constant) and total entropy $H=E+p / \rho$, where total energy $E=C_{v} T+K$ with $C_{v}$ the specific heat at constant volume, $T$ the static temperature and $K$ the kinetic energy of the flow. The molecular Prandtl number Pr is 0.72 , and the turbulent Prandtl number 
$\operatorname{Pr}_{t}$ is set 0.9 . The stress $\sigma_{i j}$ is expressed by using the Boussinesq approximation as follows:

$$
\sigma_{i j}=2\left(\mu+\mu_{t}\right)\left(S_{i j}-\frac{1}{3} S_{k k} \delta_{i j}\right)
$$

where $S_{i j}=\frac{1}{2}\left(\frac{\partial U_{i}}{\partial x_{j}}+\frac{\partial U_{j}}{\partial x_{i}}\right)$ is the strain rate tensor. The molecular viscosity $\mu$ is computed by using the Sutherland law: $\frac{\mu}{\mu_{\infty}}=\frac{1+\mathrm{T}_{s} / T_{\infty}}{T / T_{\infty}+T_{s} / T_{\infty}}\left(\frac{T}{T_{\infty}}\right)^{3 / 2}$, where $T_{S}=110.4(\mathrm{~K})$ and subscript $\infty$ indicates the free-stream property. The turbulent viscosity $\mu_{t}$ has to be modeled.

\subsection{The SED-SL transition model}

The SED theory has extended the classical mixing length concept [20] to multiple Reynolds stress lengths to describe both the Reynolds shear stresses and the Reynolds normal stresses [15]. The Reynolds shear stress length $\ell_{12}$ in a compressible TBL is defined as:

$$
\ell_{12}=\frac{\sqrt{-\overline{\rho u^{\prime} v^{\prime}}}}{\sqrt{\rho} \partial_{y} U},
$$

where $U$ is the streamwise mean velocity, $u^{\prime}$ and $v^{\prime}$ are the streamwise and wallnormal velocity fluctuations, and overline denotes Reynolds averaging. $\ell_{12}$ has been interpreted as the characteristic size of eddies responsible for the wall-normal turbulent transport of momentum. It is applied for expressing $\mu_{t}$ of TBL in the SED-SL model [17, 18, 19], as follows:

$$
\mu_{t}=\rho \ell_{12}^{2}|\omega|
$$

where $\omega=\partial_{y} U-\partial_{x} V$, following the same convention as in the Baldwin-Lomax model [21].

The SED theory predicts that the stress length $\ell_{12}$ possesses a multilayer form in fully-developed TBL $[14,15]$ :

$$
\begin{aligned}
& \ell_{12}^{+}=\ell_{12}^{+ \text {inner }}\left(y^{+}\right) \times \ell_{12}^{+ \text {outer }}(r), \\
& \ell_{12}^{+ \text {inner }}\left(y^{+}\right)=\ell_{0}^{+}\left(\frac{y^{+}}{9.7}\right)^{3 / 2}\left[1+\left(\frac{y^{+}}{9.7}\right)^{4}\right]^{1 / 8}\left[1+\left(\frac{y^{+}}{y_{b u f}^{+}}\right)^{4}\right]^{-1 / 4}, \\
& \ell_{12}^{+ \text {outer }}(r)=\frac{\left(1-r^{4}\right)}{4(1-r)}
\end{aligned}
$$

where $y^{+}$is the distance from the wall in the wall unit (i.e. $y^{+}=y u_{\tau} / \nu_{w}, \nu$ is the kinematic viscosity, $u_{\tau}$ is the friction velocity: $u_{\tau}=\sqrt{\left.\mu_{w} \frac{d U}{d y}\right|_{w} / \rho_{w}}$, and subscript $w$ denotes wall value), $y_{b u f}^{+}$is the thickness of the buffer layer, $\ell_{0}^{+}=9.7^{2} \kappa / y_{b u f}^{+}$ is the near-wall eddy length, and $\kappa$ is the Karman constant. $r=1-y / \delta$ is the 
outer coordinate, being the distance (to the wall direction) from the edge (at $\delta$ ) of the TBL. $\delta$ is defined as the thickness of $0.99 U_{e}\left(U_{e}\right.$ is free stream velocity) for flat-plate TBL. In general (e.g. the cone), $\delta$ needs to be evaluated following the Baldwin-Lomax model [21], by

$$
\delta=\frac{y_{\max }}{0.3}
$$

where $y_{\max }=$ location $\left(\max \left(\ell_{12}^{+ \text {inner }}|\omega|\right)\right)$. Eq.(7)-Eq.(9) describe a wall-normally four-layer structure for the canonical TBL, including the viscous sublayer (with thickness 9.7), buffer layer (with thickness $y_{b u f}^{+}$), log-layer and bulk/wake flow (with the $1-r^{4}$ structure); it is easy to verify that for $y^{+} \gg y_{b u f}^{+}$and $r \approx 1$ in Eq.(7), i.e. in the matching region between the inner and outer regimes of TBL, one obtains the celebrated linear law $\ell_{12}^{+} \approx \kappa y^{+}$of Prandtl [20].

For the canonical TBL the SED asserts that $y_{b u f}^{+}$is about 41 , and $\kappa, 0.45$, thus $\ell_{0}^{+}$is about 1.03. Consequently there is no free parameter in Eq.(7)-Eq.(9), but adequate to accurately predict the whole mean velocity profile of canonical TBL at all (above moderate) Re [14]. As to non-canonical TBLs, the above four-layer structure may be deformed by the influential factors (such as pressure gradient) and evolve spatially. A crucial assumption of the SED is that, because the wall constraint remains the dominant effect, the deformation is only finite, slow and continuous, such that it can be described with variable multi-layer structure parameters. Two parameters have been identified to be crucial: $\ell_{0}^{+}$and $y_{b u f}^{+}$, which determine the global size and location of the strongest eddies (which are vulnerable to environmental variation) and thus characterise the major deformation of the boundary layer. The above assumption has been validated for a series of non-canonical TBLs, in particular, the TrBL $[18,19]$.

To describe the TrBL, Xiao and She [18] has proposed a two-dimensional multiregime structure by postulating a streamwise multilayer dilation with respect to the leading edge (i.e. $x=0$ ) for $\ell_{0}^{+}$and $y_{b u f}^{+}$. Here, for the transitional HBL of cone, they are written as follows:

$$
\begin{aligned}
& \ell_{0}^{+}=\ell_{0 \infty}^{+}\left(\frac{x}{x^{*}}\right)^{5}\left[1+\left(\frac{x}{x^{*}}\right)^{10}\right]^{-1.5}\left[1+\left(\frac{x}{\beta_{l} x^{*}}\right)^{10}\right] \beta_{l}^{10}, \\
& y_{b u f}^{+}=41\left(\frac{x}{x^{*}}\right)^{5.5}\left[1+\left(\frac{x}{x^{*}}\right)^{10}\right]^{-5.5 / 10},
\end{aligned}
$$

in which three transition parameters are introduced. $x^{*}$ is called the transition center, being a newly-identified indicator for the transition location that is believed superior in physics than the conventional transition onset location [18]. $x^{*}$ requires further modeling. In the present study it is an adjustable model parameter. $\ell_{0 \infty}^{+}$ characterises the near-wall eddy size of the fully-developed TBL; it determines the skin friction and surface heat flux after the transition. $\ell_{0 \infty}^{+}$depends on pressure gradient, Ma and wall temperature, etc. [19]. For quasi adiabatic TBL with less than moderate pressure gradient, $\ell_{0 \infty}^{+}$is around unity, i.e. the canonical TBL value; for hypersonic flow over significantly cooled wall, it can be substantially smaller than 
unity owing to the compression effect on the eddy size. $\beta_{l} \geq 1$ is a coefficient that quantifies the strength of the transition overshoot (a phenomenon that the skinfriction coefficient and Stanton number at around the transition end considerably surpass their TBL values at the same Reynolds number). It is the ratio of the location where the transition begins to relax to the fully-developed TBL to the location of the transition center, thus it (when larger than unity) defines a transition regime between the laminar and fully-turbulent regions. The larger $\beta$, the wider the transition regime, the stronger the transition overshoot, and the higher the peak heat flux. When $\beta=1$, no transition regime or transition overshoot appears. For most by-pass transitions, the typical value of $\beta$ is about 1.1.

Here we apply the above model to investigate the hypersonic transitional flows over straight cones at moderate AoA. As is presented, varying the three model parameters yields accurate descriptions of a substantial variety of skin friction and surface heat flux distributions in the HBL transition of straight cone. A note about the computation is that, the streamwise coordinate $x$ in Eqs. 11 and 12 is approximated by the axial coordinate of the cone (which is also denoted by $x$ with $x=0$ being the cone nose tip) for computational simplicity, which affects very little at moderate AoA.

\subsection{Numerical implementation}

The RANS equations closed by the SED-SL model has been solved by using the CFL3D, which was developed in the early 1980s in the Computational Fluids Laboratory at NASA Langley Research Center, and has been used to support numerous NASA programs since then and continues to be used today, particularly for validation and verification of newly developed turbulence models. The current SED-SL code has been developed based on the code of the B-L model in CFL3D, considering their apparent similarity.

Three sets of grid have been tested for the $7^{\circ}$ half-angle cone. Grid one has a uniform mesh with grid number 31 in the circumferential direction (from $0^{\circ}$ to $180^{\circ}$, i.e. a half-model simulation), an increasingly coarsening mesh with grid number 173 in the axial direction, and a stretching mesh with grid number 131 in the wall normal direction. It has been verified that the first wall-normal mesh has a dimension of $y^{+}<0.7$ over the whole cone surface for all the investigated cases. Grid two is doubled in the circumferential direction, and grid three is doubled in both the circumferential and axial directions, for testing grid convergence.

The boundary conditions are set as the following. The free stream boundary condition is used for the inflow. The isothermal, no-slip, solid-wall boundary condition is employed on the cone surface. Since we have conducted a half-model simulation, the symmetry condition is applied on the symmetry plane. Finally, the extrapolation condition is used for the outflow.

As to the numerical algorithms, an implicit approximate-factorization method is applied for time advancing. The viscous fluxes are computed with the secondorder central differences, and the inviscid fluxes are computed with the upwind flux-difference-splitting method. The RANS equations are solved in parallel on a workstation with $16 \mathrm{CPU}$ cores. 
The experimental database used for validation are listed in table 1. They are selected from the very recent measurements on the surface heat flux and/or surface temperature of $7^{\circ}$ half-angle straight cones at moderate AoA, all covering a major portion of cone surface with high measurement accuracy. The first experiment was conducted by Willems et al. [22] in the hypersonic wind tunnel (H2K) of the German Aerospace Center (GAC) in Cologne. Surface temperatures and heat fluxes were measured by means of quantitative infrared thermography for the whole cone surface. The second experiment was performed by Chen et al. [23] in the $\Phi 1 \mathrm{~m}$ Hypersonic Wind Tunnel of the China Aerodynamics Research and Development Center (CARDC). Only surface temperature was measured for the windward and leeward cone surfaces by quantitative infrared thermography. In table $1, \mathrm{R}$ denotes cone nose-tip radii, $P_{0}$ is total pressure, $T_{0}$ is total temperature, and $T_{w}$ is the cone surface temperature set in the current numerical simulation.

Table 1 The flow parameters of the test cases

\begin{tabular}{cccccccc}
\hline Case & $R e_{\infty}\left(\times 10^{7} m^{-1}\right)$ & $M a_{\infty}$ & $P_{0}(\mathrm{mPa})$ & $T_{0}(\mathrm{~K})$ & $T_{w}(\mathrm{~K})$ & $\mathrm{AoA}\left({ }^{\circ}\right)$ & $\mathrm{R}(\mathrm{mm})$ \\
\hline GAC-0,6 & 0.911 & 7 & 2.288 & 601.4 & 300 & 0,6 & 1.6 \\
CARDC-0,2,4,6 & 1.0 & 6 & 1.1 & 474 & 300 & $0,2,4,6$ & 0.05 \\
\hline
\end{tabular}

\section{Result}

Before presenting the results, the effects of the grid and isothermal wall condition on the simulation results have been assessed. For the grid convergence, it has been found that grid one is adequate for AoA no more than $4^{\circ}$, but grid two has to be used for accurately calculating the surface heat flux around the leeward center of the cone at $A o A=6^{\circ}$, because of the strong streamwise vortices there. The other issue is about the isothermal wall condition applied in the simulation, which does not agree with the experimental setting. In the GAC experiment, the cone surface temperature was measured by quantitative infrared thermography and the surface heat flux was derived accordingly based on a one-dimensional heat-transfer model. One finds in [22] that for the GAC cases the surface temperature in the turbulent region is clearly larger than that in the laminar region, and the temperature difference can be as large as $60 \mathrm{~K}$ for the GAC- 6 case, which is about $10 \%$ of the stagnation temperature (whereas in the CARDC-6 experiment, only about 1\%). So the cone surface is not actually isothermal as the setting of the numerical simulation. We have assessed the effects of the isothermal wall condition on the surface heat flux distribution by increasing the wall temperature from $300 \mathrm{~K}$ to $340 \mathrm{~K}$ in the GAC-6 case. Indeed, about $15 \%$ decrease of heat flux can be found in some locations. Neglecting such a discrepancy, the isothermal wall condition is utilized for simplicity in the current studies.

Validation of the model is conducted by comparing the surface heat fluxes. The dimensionless heat flux $C_{h}$ (or the Stanton number $S t$ ) is defined as:

$$
C_{h}=S t=\frac{\left.k_{w} \frac{\partial T}{\partial y}\right|_{w}}{\rho_{\infty} U_{\infty} C_{p}\left(T_{r}-T_{w}\right)},
$$

where $T_{r}=T_{\infty}\left(1+r M a_{\infty}{ }^{2} / 5\right)$ with $r=\sqrt{P r}, k$ is thermal conductivity, and $C_{p}$ is the specific heat at constant pressure. The focus on $C_{h}$ is because of the following. 
$C_{h}$ is the most widely measured quantity and the upmost concern in engineering applications. Besides, the location, strength and extent of hypersonic boundary layer transition, which have been puzzling to modelers and engineers and enduring numerous studies for decades, can entirely be manifested by the $C_{h}$ distribution. It also provides a strong constraint to the flow field: in case that the $C_{h}$ distribution is correctly predicted, the flow is very likely correct.

The current study serves a purpose to validate the SED-SL description of the various hypersonic transitional boundary layers over the cone through the aforementioned three model parameters (i.e. $x^{*}, \ell_{0 \infty}^{+}, \beta_{l}$ ), which are determined after a prediction-correction procedure: first, setting the parameter values; second, solving the RANS equations with the SED-SL model; third, comparing the numerical prediction with the experimental results and updating the parameters until an accurate prediction is achieved. Note that such a procedure can easily be implemented only because the current model parameters have clear physical meanings and directly related to the shape of the $C_{h}$. By continuously repeating this procedure in different sample flow cases, the SED-SL eventually will cover a wide flow regime and gain full prediction capacity for engineering applications.

\subsection{Simulation of the GAC Experiment}

Following the above procedure, the three parameters are measured for the GAC and CARDC cases. For the current flow cases $\ell_{0 \infty}^{+}$is found invariant with AoA and the circumferential angle $\theta$, and identical to the canonical TBL value $(\approx 1.0)$, which perhaps is because the Mach number is not very large and the cone surface is not very cold. In comparison, $\beta_{l}$ and especially $R e_{x^{*}}$ vary with AoA and $\theta$, as shown in Figure 1.

$R e_{x^{*}}$ is a new indicator of the transition front. At $A o A=2^{\circ}$ and $4^{\circ}$ of the CARDC cases (not shown in Fig. 1a for clearance), $R e_{x^{*}}$ varies almost linearly with $\theta$ and becomes flattened near $\theta=0^{\circ}$ and $180^{\circ}$ to adapt to the symmetry condition, which is as expected because small AOA has only a perturbation effect on the transition. At $A o A=6^{\circ}$, one finds in Figure 1a that $R e_{x^{*}}$ begins to break up into three regions: a windward region with a significantly postponed and rapid changing transition front, a wide cross-flow region with a relatively slowly advancing transition front, and a narrow leeward region with a much earlier transition front. Such a behaviour is more apparent for the GAC-6 case than the CARDC-6 case. The reason for this break-up is likely because of the circumferential variation of the dominant transition mechanism at moderate AoA. Indeed, the Mack second mode that governs the whole cone surface at zero incidence shrinks to a portion of the windward region with increasing AoA. The cross-flow mode occupies the main surface of the cone owing to the wide-spread pressure gradient in the circumferential direction, leaving only a narrow leeward region for the streamwise vortex mode, where streamlines converge and form counter-rotating streamwise vortices before separation happens at larger $A \circ A$ (e.g. $A o A>9^{\circ}$, [22]).

The variation of $\beta_{l}$ is simpler (Figure $1 \mathrm{~b}$ ). It is invariant with AoA and $\theta$ in the cross-flow and leeward regimes, but decreasing (linearly, in the present empirical setting) with decreasing $\theta$ in the windward region. In the cone windward, $\beta_{l}$ also decreases with increasing AoA, which means that the transition overshoot is relatively diminishing in the cone windward as the AoA is increased, owing perhaps 
to the stronger compressibility there. More studies are needed to clarify this phenomenon. In addition, there is a clear difference regarding the transition overshoot strength between the GAC-6 and CARDC-6 cases. The CARDC-6 case has much stronger transition overshoots than those of GAC-6. The reason is unclear, but there might be a difference between the tunnel noise levels of the CARDC wind tunnel and the GAC wind tunnel [24]. 
Figure 2 The $S t$ distribution along the meridian line computed by the SED-SL model for the GAC-0 case, compared with the measured $S t$ distribution and the measured surface temperature increment with appropriate normalization to collapse in the laminar region.

and the peak surface heat flux are computed very accurately, whose relative errors are less than $2 \%$.

Figure 2 also includes a comparison between the $S t$ distribution and the measured surface temperature increment $\left(\Delta T_{w}\right.$, with a reference $T_{w}$ of $\left.300 \mathrm{~K}\right)$ distribution with proper normalization. The reason for this comparison is explained as the following. Hypersonic boundary layer transition has been more and more frequently diagnosed by quantitative infrared thermography for its high fidelity, wide angle of view and nonintrusion. However, the technique measures only the surface temperature and the heat flux has to be derived accordingly by an algorithm with specific heat transfer assumptions. In the CARDC experiments (and many others), only the surface temperature data are provided. Thus it is interesting to study the relationship between $\Delta T_{w}$ and $S t$, to identify whether or not $\Delta T_{w}$ can be an acceptable approximation of the surface heat flux distribution. In Figure 2 one finds that, for the GAC-0 case, by introducing a proportional factor to collapse $\Delta T_{w}$ with $S t$ in the laminar region, the rescaled $\Delta T_{w}$ almost collapses with $S t$ over the whole transitional boundary layer, except a rather limited distinction owing mainly to the discrepancy of the transition onset location. Although this apparent similarity has to be further validated with heat transfer models, it is understandable because the measurements are often conducted with a very short duration, such that the surface temperature increment is strongly correlated with the local surface heat flux. Therefore, in the next section, the CARDC data of surface temperature increment are utilized as substitutes for studying the heat flux distributions, which is more convincing considering their temperature increments are much smaller than those of the GAC cases.

Figure 3 compares the SED-SL computed St contour with the measured one for the GAC-6 case. The simulation result is almost identical to the measured contour over the whole cone surface. A unique behaviour in the experiment is that, there is an array of distinct, standing cross-flow vortices at around $\theta=135^{\circ}$, extending streamwise over a considerably long length with a slight inclination towards the 
leeward side of the cone. These vortices are stationary, usually occur in a quiet or low-noise environment, and are often induced by surface roughness [10]. The crossflow vortices disappear suddenly at $\theta<135^{\circ}$, which is due to different wind-tunnel runs, as said in [22]. So, it is clear that a large portion of the cone surface is governed by the cross-flow transition, as aforementioned. In a rather narrow range around $\theta=180^{\circ}$, the transition onset is significantly earlier, along with a heat flux valley at $\theta=180^{\circ}$, which is apparently due to the counter-rotating streamwise vortices. As shown in Figure 3, the SED-SL simulation has reproduced with many details the elaborate distribution of $S t$ on the cone surface, but dose not predict the standing cross-flow vortices on the lateral side of the cone.

The reason deserves further discussions. The current model is based on describing the dilation symmetry property of boundary layers, which emerges as a result of the self-organization of a set of turbulent eddies. In case of a flow dominated by distinct vortices, which often happens when the disturbances are slight or particular, the flow evolves dynamically with usually discrete characteristic lengths and unique spatial distribution, rather than statistically with continuously-distributed length scales (such that the power law is established). Therefore, it will be more difficult, if not possible, for the SED-SL to describe the flows whose length scales are not fully agitated, e.g. the early stage of transition in some cases. In the hypersonic transition, such flows could be the stationary cross-flow vortices, or particular development of the Mack second mode that may lead to an additional peak heat flux before transition $[25,26]$.

More quantitative comparisons are conducted by plotting the SED-SL computed St profiles along different meridian lines with together the corresponding measured ones acquired from the experimental contour (i.e. Figure 3a), which are shown in Figure 4 for the windward, cross-flow and lee sides, respectively. For all the 
circumferential angles, the simulated profiles agree excellently with the measured ones over the whole hypersonic transitional boundary layers. The relative errors of the computed transition onset location and peak heat flux are within a few percent, which are prominent if one recalls the current status of the hypersonic transition models.

Let us discuss more about the transition overshoot. Transition overshoot widely occurs in by-pass transitions with strong disturbances. It results in significant increase of peak surface heat flux in hypersonic flows that affects the thermal protection design. A clear understanding of the overshoot phenomenon is not yet established, which has attributed to the failure of most RANS-based transition models in predicting the peak heat transfer. Qin et al. [27] have used an algebraic intermittency factor to accelerate the development of TBL in the late transition region, such that the overshoot phenomenon is reproduced with a reasonable degree of accuracy. However, Qin et al.'s method is based on the perspective of transition model construction, and its applicability to complex configurations requires further investigations [13]. In the present approach, the transition overshoot is characterized by a single parameter, $\beta_{l}$, which, if larger than unity, introduces a streamwise transition layer in between the laminar and full-turbulent flows. In this layer, eddies stimulated upstream by intense disturbances become highly developed, leading to a violent transition organization that requires a considerable length (quantified by $\beta_{l}$ ) to relax eventually to a traditional TBL. The streamwise transition layer mimics the buffer layer (where eddies are strong) in the wall-normal direction of TBL, which exists in between the viscous sublayer and the full-developed log layer and bulk flow [28]. From this point of view, the transition overshoot is a normal phenomenon, and the robustness of $\beta_{l}$ in depicting various by-pass transitions is understandable.

Now we report the streamwise development of the hypersonic transitional boundary layer on the cone surface revealed by the three physical parameters of the SED-SL model. Figure 5 shows the variations of $\ell_{0}^{+}$and $y_{b u f}^{+}$along different meridian lines for the GAC- 6 case. $y_{b u f}^{+}$denotes the thickness of the buffer layer, i.e. the location of the near-wall coherent structures, which has crucial impact on the shape of the mean velocity profile, but is difficult to measure in experiments on high-speed boundary layers. In the current setting of the SED-SL model, $y_{b u f}^{+}$has a streamwise two-layer development that depends only on $x^{*}$ (see Eq. (12) and Figure 5b), which can be seen as a first-order approximation. With DNS and possible experimental profile data, the two-layer model of $y_{b u f}^{+}$can be assessed and refined in the future.

On the other hand, $\ell_{0}^{+}$is more directly relevant to the skin friction and surface heat flux distributions on the cone surface because it determines the magnitude of the stress length and thus the levels of the eddy viscosity and eddy conductivity. As shown in $5 \mathrm{a}, \ell_{0}^{+}$possesses a three-layer streamwise evolution, especially for the crossflow and leeward sides of the cone (on the lee side $\theta=174^{\circ}$ is selected to avoid the heat flux valley), where about $25 \%$ overshoot can be found for $\ell_{0}^{+}$comparing with the TBL value. At the windward center, the transition overshoot is not apparent as aforementioned. From Figure 5 one finds that, although the transition mechanisms are very different in the windward, cross-flow and leeward sides of the cone, the boundary layer development follows a same way, with only quantitative difference on $\ell_{0}^{+}$. 


\subsection{Simulation of the CARDC experiment}

The measurement conducted by Chen et al. in CARDC [23] is a very recent experimental study about the hypersonic transitional flow on a sharp cone. The data are of high accuracy, high resolution, and high repeatability. However, only surface temperature increment data are provided, and the measurement is restricted to the windward and leeward sides of the cone, making the cross-flow side inaccurate owing to the large angle of view. Here, we present the computation of the CARDC cases with the SED-SL transition model.

The settings of the model parameters for the CARDC cases are shown in figure 1. Because the surface temperature increment is closely correlated with the Stanton number, as revealed by figure 2, we directly compare the simulation results of $S t$ with the rescaled temperature increment $\Delta T_{w}$, in which the proportional factor is determined by making the laminar data collapse together.

Figure 6 compares the rescaled $\Delta T_{w}$ contours with the SED-SL computed $S t$ contours for all the four CARDC cases. For the cones with nonzero AoA, the windward and leeward surfaces are compared separately. The agreement between the simulation and measurement is excellent, throughout the cone surface and for all test cases. Therefore, the current model demonstrates a high accuracy and a wide adaptivity in describing various hypersonic transitional boundary layers.

Further comparisons are performed in figures 7-10 for each CARDC case, which confirm the agreements of the contours. In figure 7, specifically, the $S t$ profile along the cone axial direction computed by the SED-SL is compared with the rescaled $\Delta T_{w}$ profile measured in the experiment for the CARDC- 0 case. Also plotted in figure 7 is the GAC- 0 case, to evaluate the similarity and discrepancy between the two experiments. One finds that the SED-SL model accurately describes the surface heat transfer properties of both the two transitional boundary layers. A major difference between the two flows is the transition onset location (or $x^{*}$ ), which is earlier in the CARDC-0 case. Besides the small discrepancy on Ma, two reasons might contribute to this difference. First, the nose-tip radii of the GAC cone is much larger than that of the CARDC cone, leading to a postponed transition location for the GAC-0 case [3]. Second, the freestream noise level characterized by $T u=p_{r m s} / p$ is about $3.9 \%$ for the CARDC-0 case according to [23], and that of the GAC-0 case is about $2 \%$, which also result in an earlier transition for the CARDC-0 flow [29]. Other than the transition onsets, the two transitional flows are almost identical in the laminar and fully turbulent regimes, showing the reliability of the SED-SL model.

\section{Discussion and conclusion}

In this work the HBL transition on a slender cone at moderate incidence has been studied via a symmetry-based length model: the SED-SL model. It is shown that, by properly setting the multi-regime structure through only three parameters (i.e. a transition center, an after-transition near-wall eddy length, and a transition width coefficient related to overshoot strength), the SED-SL correctly describes the heat flux distribution on the whole cone surface, agreeing closely with multiple sets of wind tunnel data. The results demonstrate the validity of the SED theory regarding the multi-regime similarity across a non-equilibrium transitional process, which is 
an organizational principle for TBL (due to wall dilation symmetry), and then yields a simple algebraic model for transitional HBLs.

The current SED-SL model has three distinct features compared to popular RANS models: its simplicity, accuracy and transparency for its parameter interpretation. The goal of this paper is not yet to conclude a matured transition model for industrial application, but to validate the symmetry-based description of the transitional HBLs. The current success indicates that, with the accumulation of validated flow cases, a practical transition model will emerge for computing a considerably large number of flows, with both high prediction accuracy and wide adaptivity.

Note that the "similarity structure" proposed by the SED theory is statistical, and is distinct from those highly unstable patterns such as instantaneous vortex structures or wave-like perturbations (which have been the targets in the stability theory and coherent structure studies). But we assert that only the similarity structure is stable and is directly related to mean flow properties (such as skin friction), so as to be relevant to engineers' prediction ability. So, it would become the most important task to characterize and even quantify this similarity structure for complex engineering boundary layer flows.

For future perspective, it would be intriguing to investigate the dependence of the transition-relevant multilayer structure parameters on various flow parameters. For example, our preliminary study shows that $\ell_{0}^{\infty}$ decreases with increasing $M a$, which is sound as compressibility yields a "compression" of eddy size globally, hence a smaller eddy-viscosity. Another crucial transition parameter is the transition center $x^{*}$, which defines the transition location that is of utmost importance in aerospace engineering. New correlation relationships between $R e_{x^{*}}$ and multiple transition influential factors have to be established for the current SED-SL model to reach full predictability, which we will demonstrate to be feasible in a future communication, since we have captured the right similarity structure.

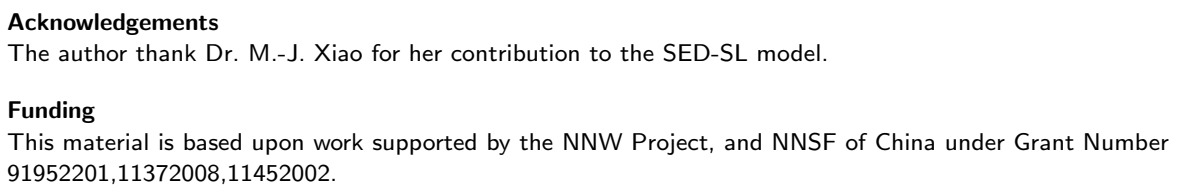




\section{References}

1. Anderson, J.D.: Hypersonic and High Temperature Gas Dynamics. McGraw-Hill Book Company, UK (1989)

2. Schneider, S.P.: Hypersonic laminar-turbulent transition on circular cones and scramjet forebodies. Prog. Aerospace Sci. 40, 1-50 (2004)

3. Paredes, P., Choudhari, M.M., Li, F., s. Jewell, J., Kimmel, R.L., Marineau, E.C., Grossir, G.: Nose-tip bluntness effects on transition at hypersonic speeds. J. Spacecraft Rockets 56, 369-387 (2019)

4. Menter, F.R., Langtry, R., VIker, S.: Transition modelling for general purpose cfd codes. Flow Turbul. Combust. 77, 31-48 (2006)

5. Menter, F.R., Smirnov, P.E., Liu, T., Ravikanth, A.: A one-equation local correlation-based transition model Flow Turbul. Combust. 95, 583-619 (2015)

6. Savill, A.M.: Evaluating turbulence model predictions of transition. Appl. Sci. Res. 51, 555-562 (1993)

7. Durbin, P.A.: Some recent developments in turbulence closure modeling. Annu. Rev. Fluid Mech. 50, 77-103 (2018)

8. Lee, C.-B., Jiang, X.-Y.: Flow structures in transitional and turbulent boundary layers. Phys. Fluids 31, 111301 (2019)

9. Chen, J.Q., Yi, S.H., Li, X.L., Han, G.L., Zhang, Y.F., Yang, Q., Yuan, X.X.: Theoretical, numerical and experimental study of hypersonic boundary layer transition: Blunt circular cone. Appl Thermal Engineering 194 116931 (2021)

10. Wan, B.B., Tu, G.H., Yuan, X.X., Chen, J.Q., Zhang, Y.F.: Identification of traveling crossflow waves under real hypersonic flight conditions. Phys Fluids 33, 044110 (2021)

11. Li, X.H., Chen, J.Q., Huang, Z.F., Yang, Q., Xu, G.L.: Stability analysis and transition prediction of streamwise vortices over a yawed cone at mach 6. Phys Fluid 32, 124110 (2020)

12. Wang, L., Fu, S.: Modelling flow transition in a hypersonic boundary layer with reynolds-averaged navier-stokes approach. Sci. China Phys. Mech. Astron. 52, 768-774 (2009)

13. Liu, Z.J., Yan, C., Cai, F.J., Yu, J., Lu, Y.H.: An improved local correlation-based intermittency transition model appropriate for high-speed flow heat transfer. Aerospace Sci. Technol. 106, 116122 (2020)

14. She, Z.S., Chen, X., Hussain, F.: Quantifying wall turbulence via a symmetry approach: a lie group theory. J. Fluid Mech. 827, 322-356 (2017)

15. Chen, X., Hussain, F., She, Z.S.: Quantifying wall turbulence via a symmetry approach. part 2. reynolds stresses. J. Fluid Mech. 850, 401-438 (2018)

16. Chen, X., Hussain, F., She, Z.-S.: Non-universal scaling transition of momentum cascade in wall turbulence. J. Fluid Mech. 871, 2 (2019)

17. She, Z.S., Zou, H.Y., Xiao, M.J., Chen, X., Hussain, F.: Prediction of compressible turbulent boundary layer via a symmetry-based length model. J. Fluid Mech. 857, 449-468 (2018)

18. Xiao, M.J., She, Z.S.: Symmetry-based description of laminar-turbulent transition. Sci. China Phys. Mech. Astron. 62, $994711(2019)$

19. Xiao, M.J., She, Z.S.: Precise drag prediction of airfoil flows by a new algebraic model. Acta Mech. Sinica $\mathbf{3 6}$, 35-43 (2020)

20. Prandtl, L.: Uber die ausgebildete turbulenz. ZAMM 5, 136-139 (1925)

21. Baldwin, B., Lomax, H.: Thin-layer approximation and algebraic model for separated turbulent flows. In: 16th Aerospace Sciences Meeting, p. 257 (1978)

22. Willems, S., Gulhan, A., Juliano, T.J., Schneider, S.P.: Laminar to turbulent transition on the hifire-1 cone at mach 7 and high angle of attack. In: AIAA Sci. Tech. Forum, pp. 2014-0428 (2014)

23. Chen, J.F., Ling, G., Zhang, Q.H., Xie, F.T., Xu, X.B., Zhang, Y.F.: Infrared thermography experiments of hypersonic boundary-layer transition on a 7 degree half-angle sharp cone. J Exp. Fluid Mech. 34, 60-66 (2020)

24. Casper, K.M., Johnson, H.B., Schneider, S.P.: Effect of freestream noise on roughness-induced transition for a slender cone. J Spacecraft Rockets 48, 406-413 (2011)

25. Sivasubramanian, J., Fasel, H.F.: Direct numerical simulation of transition in a sharp cone boundary layer at mach 6: fundamental breakdown. J. Fluid Mech. 768, 175-218 (2015)

26. Zhu, Y.D., Chen, X., Wu, J.Z., Chen, S.Y., Lee, C.B., Hak, M.G.-e.: Aerodynamic heating in transitional hypersonic boundary layers: Role of second-mode instability. Phys. Fluids 30, 011701 (2018)

27. Qin, Y.P., Yan, C., Zhao, Z.H., Wang, J.J.: An intermittency factor weighted laminar kinetic energy transition model for heat transfer overshoot prediction. Int. J. Heat Mass Transf. 117, 1115-1124 (2018)

28. Wu, X.H., Moin, P., Wallace, J.M., Skarda, J., Duran, A.L., Hickev, J.P.: Transitional-turbulent spots and turbulent-turbulent spots in boundary layers. PNAS 114, 5292-5299 (2017)

29. Schneider, S.P.: Effects of high-speed tunnel noise on laminar-turbulent transition. J. Spacecraft Rockets 38 , 323-333 (2001) 


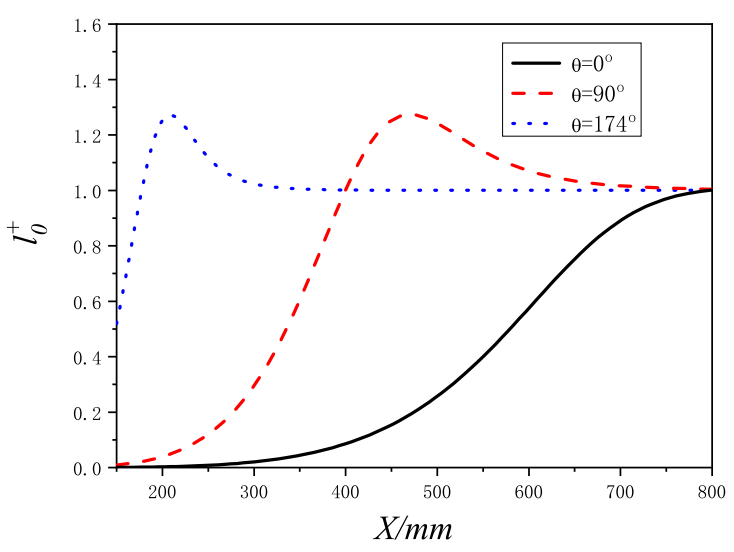

(a)

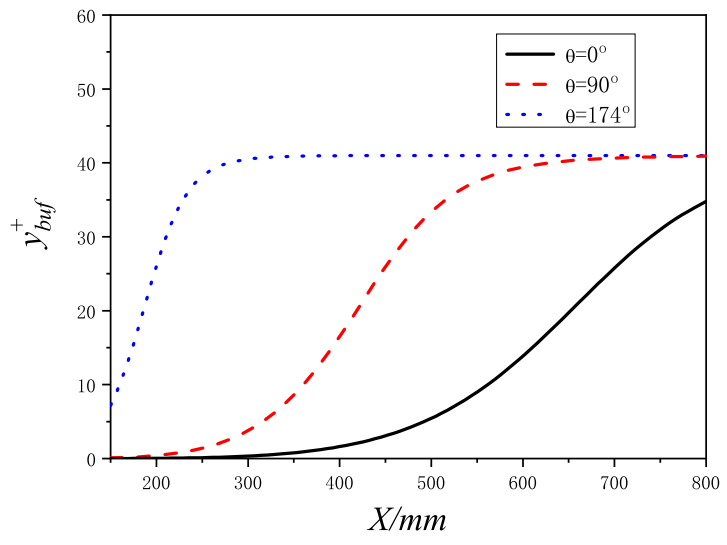

(b)

Figure 5 Evolution of (a) the near wall eddy length $\ell_{0}^{+}$and (b) the buffer layer thickness $y_{b u f}^{+}$ along different meridian lines for the GAC- 6 case 

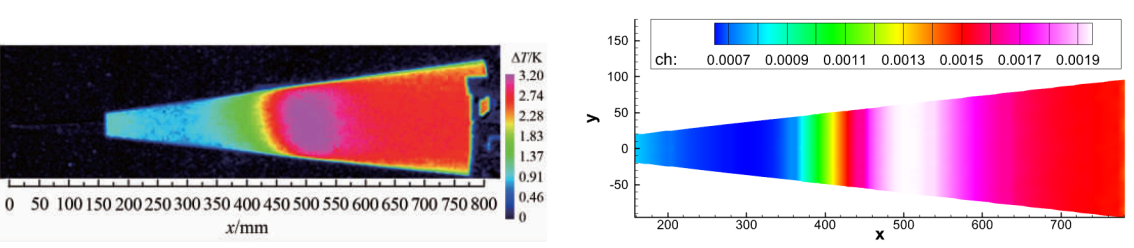

(a)
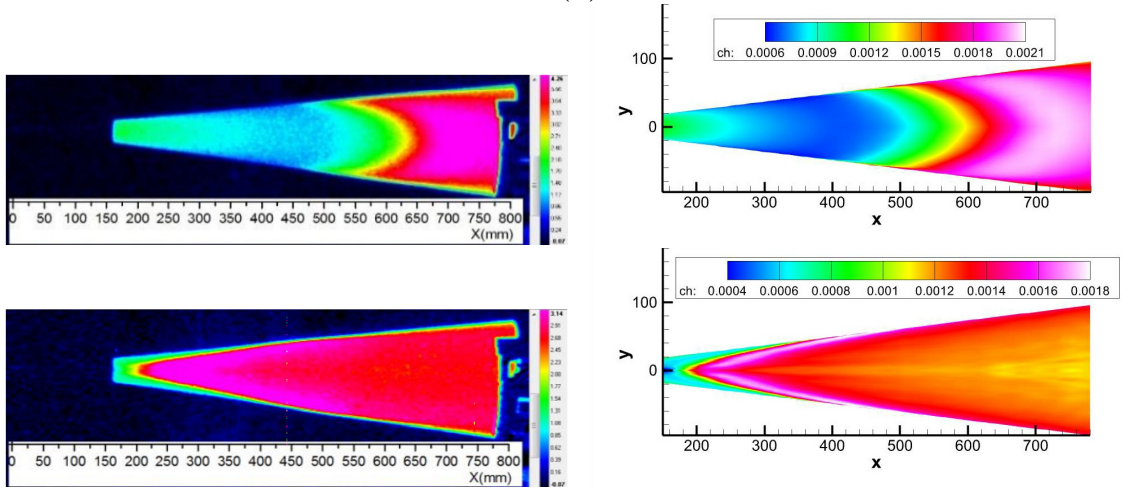

(b)
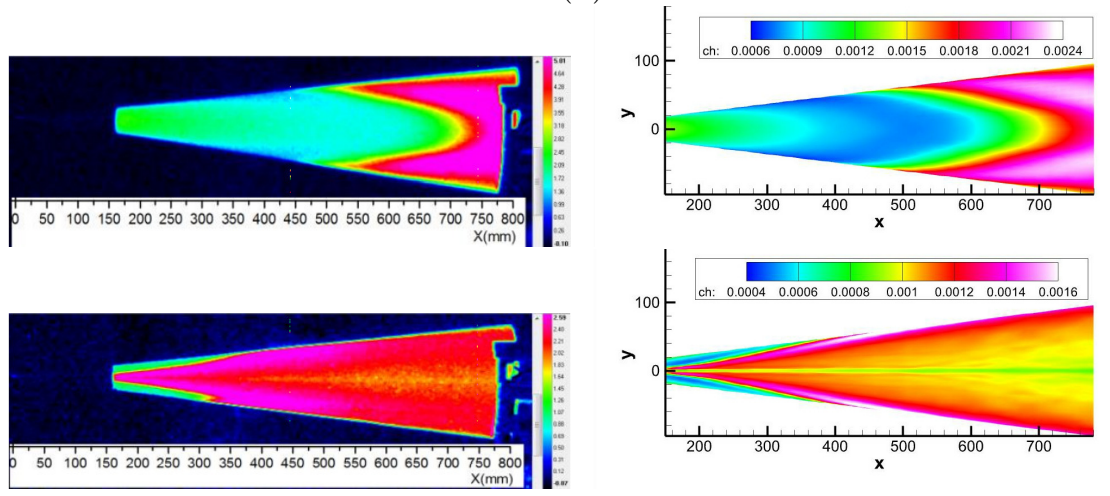

(c)
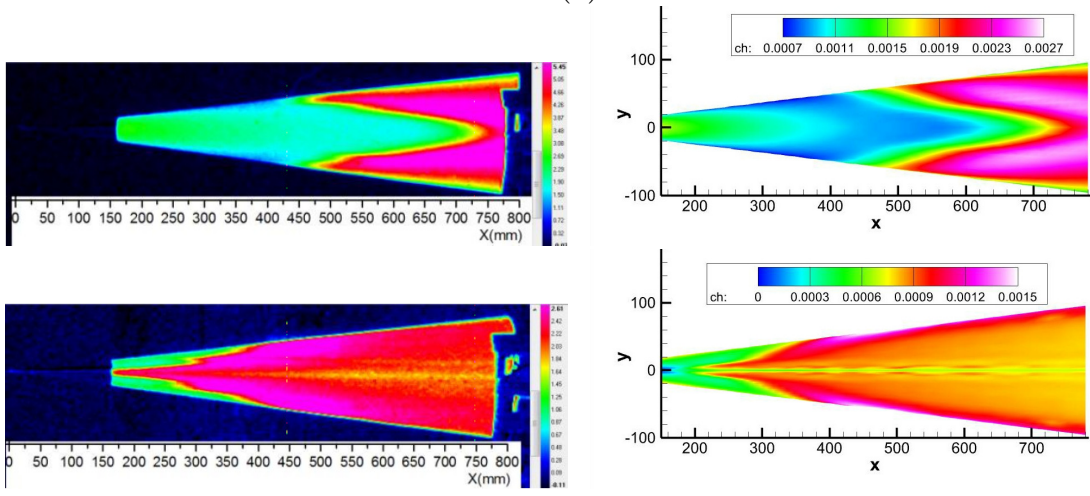

(d)

Figure 6 Comparisons between the surface temperature increment contours measured in experiment [23] and the $S t$ contours computed by the SED-SL model for (a) the CARDC-0 case, and the windward and leeward surfaces of (b) the CARDC-2 case, (c) the CARDC-4 case, and (d) the CARDC- 6 case. 


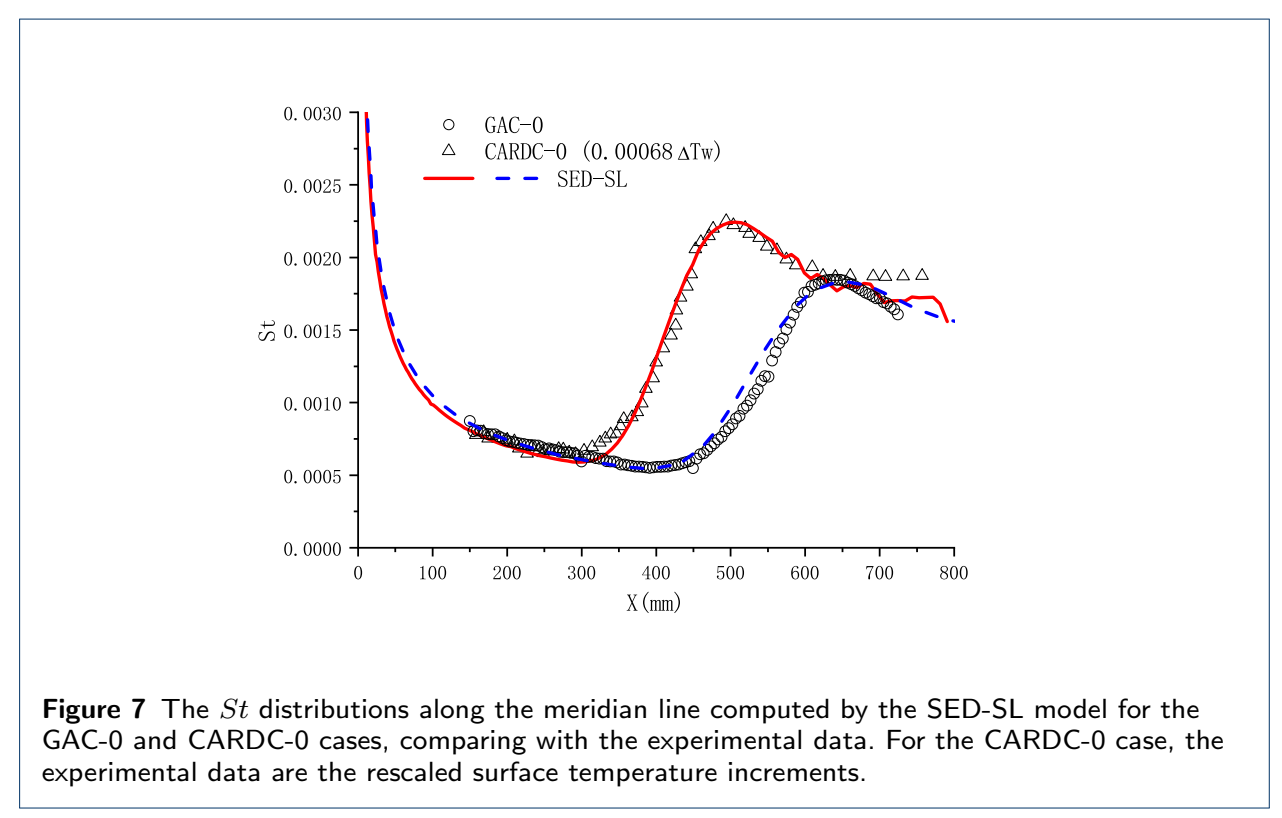




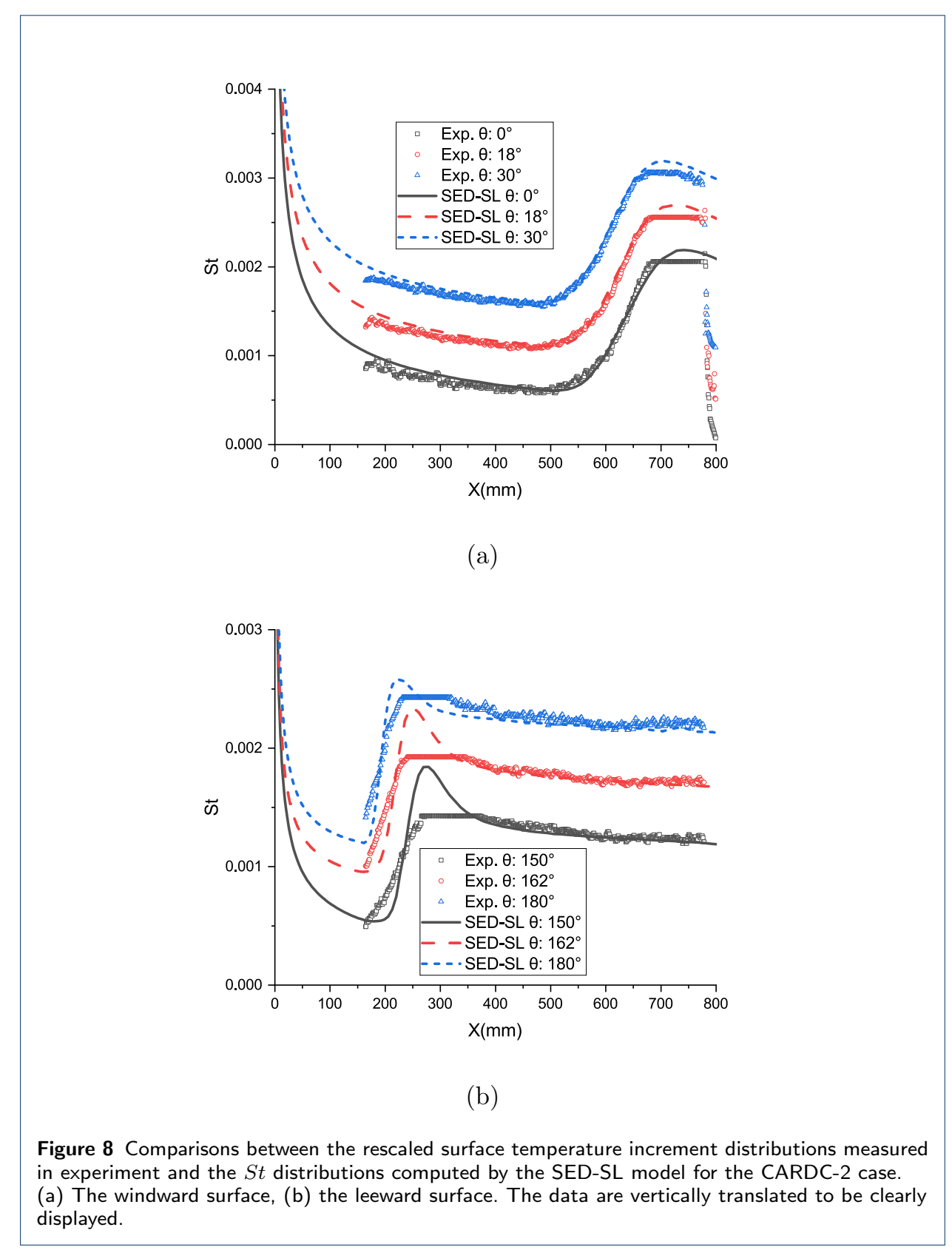




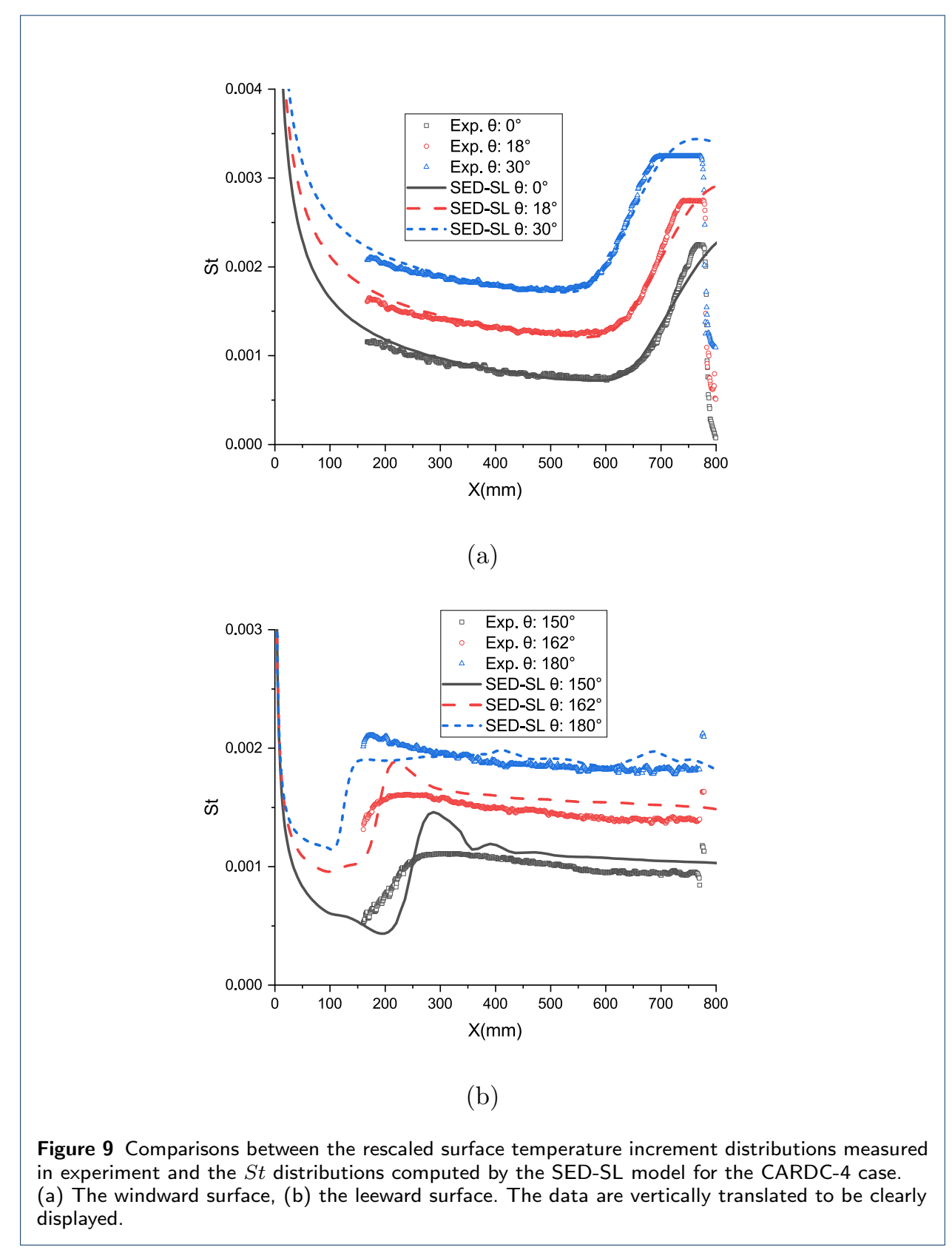




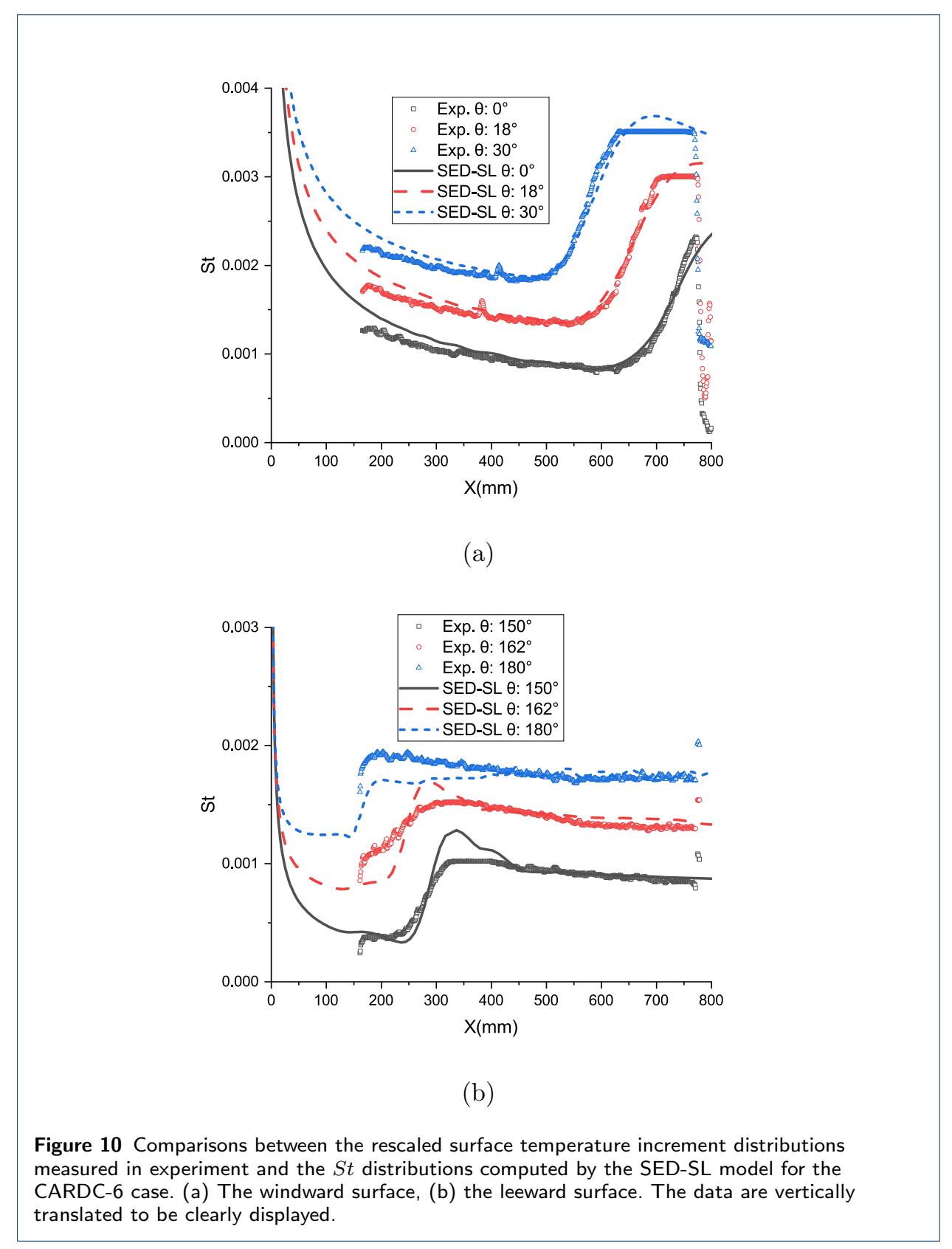

